\title{
Viterbi-based Decoding Algorithm for DBO-CSS
}

\author{
Sang-Hun Yoon, Jun-Mo Jung, Member, KIICE
}

\begin{abstract}
Differential detection algorithm for DBOCSS based on maximum signal energy detection (MSED) using viterbi algorithm is proposed. In order to mitigate SNR degradation caused by differential decoding, a modified viterbi algorithm with so called correlation metric $(\mathrm{CM})$ in every state is proposed. It is shown that the performance gain of the proposed algorithm when compared with that of the conventional differential detection with the block decoding algorithm is about $2.5 \mathrm{~dB}$ at $\mathrm{BER}=10^{-5}$.
\end{abstract}

Index Terms - viterbi, DBO-CSS, differential decoding

\section{INTRODUCTION}

RECENTLY, as the concept of ubiquitous is generalized, a lot of candidates for the terminals of sensor network have appeared by many engineers. As a standard for the candidates of the terminals, IEEE802.15.4a group selected two communication methods in October 2006. One of both methods is IR-UWB and the other is ISM DBO-CSS as they have been announced in [1].

As described in the name DBO-CSS (Differentially BiOrthogonal Chirp Spread Spectrum), DBO-CSS spreads differentially encoded bi-orthogonal codes with chirp signals as in [2-3]. Bi-orthogonal code table $\boldsymbol{C}$ is described in [1]. Because the DBO-CSS is designed for WPAN(wireless personal area network), it adopts differential modulation method which can be modulated and demodulated with very simple hardware in order to meet the requirements of WPAN; that is low-power consumption and low complexity.

But, inherently, the BER performance of differential demodulation method is worse than that of differentially encoded coherent demodulation method. When the application systems need high performance receiver, therefore the algorithms to enhance the BER performance, such as DF-DPD(decision feedback differential phase detection) in [3] and viterbi$\mathrm{DD}$ (viterbi differential detector) in [4], are usually used

\footnotetext{
Manuscript received October 1, 2011; revised October 17, 2011; accepted October 28, 2011.

Sang-Hun Yoon is with Sensor Interface Research Team, ETRI, Daejeon, Korea (Email: shyoon11@etri.re.kr), Jun-Mo Jung (Corresponding Author) is with the Department of Electronics Engineering, Kunsan National University, Kunsan, Korea(Email: jmjung@kunsan.ac.kr)
}

to minimize the performance gap between differential and coherent demodulations.

However, in case of the DBO-CSS which decodes biorthogonal code using differentially demodulated data, it is difficult to get performance enhancement with DF-DPD and viterbi-DD these two methods make hard decision results, because the hard decision degrades the coding gain of bi-orthogonal code. To solve the hard decision problem and to improve the BER performance, we propose a new decoding algorithm.

\section{PROPOSED ARCHITECTURE}

Although the DBO-CSS is proposed for low power consuming and small sized sensors, the servers which collect information from distributed sensors with very low SNR must have high performance receiver exactly to decode in such a bad communication conditions.

Viterbi algorithm is a well-known algorithm which can make maximum likelihood detection, and it is used in many applications. In this paper we have modified the viterbi algorithm to get maximum likelihood detection in DBO-CSS decoding.

\section{A. Correlation metric (CM)}

Because the DBO-CSS transmits the differentially modulated signals after bi-orthogonal coding, the receivers for DBO-CSS generally perform differential demodulation first and bi-orthogonal decoding next, in the reverse order of transmitter. In this case, the DF-DPD and the viterbi-DD algorithms can be considered to reduce the performance gap between differential and coherent demodulations. But, because such algorithms make outputs as hardly decided bits, the SNR gain of entire DBO-CSS system can't be earned.

In this paper, we intended to perform bi-orthogonal decoding first and then differential demodulation next in order to maintain bi-orthogonal coding gain. Differential modulation of in-phase and quad-phase can't maintain the bi-orthogonal relations as in figure 1 . 


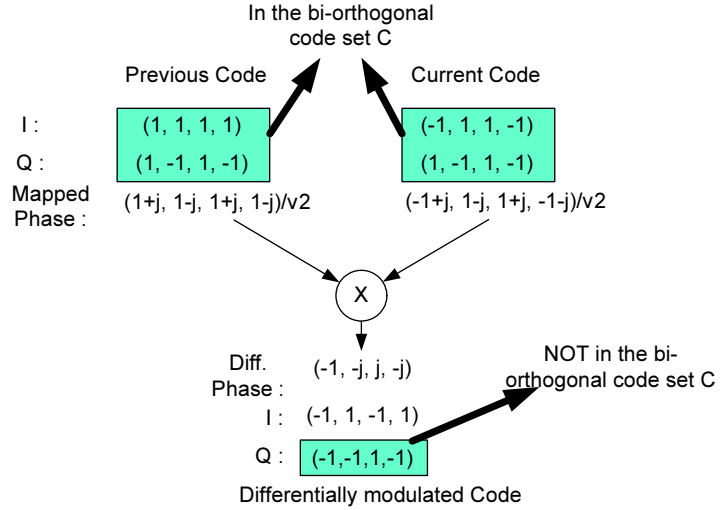

Fig. 1. Case of non-biorthogonal differential code

So, the total number of receivable code set is $4^{4}=256$, because the possible digits in every position are 4 as $\{1$, $-1, j,-j\}$. Among these code sets, there are inseparable code sets when signal phases are rotated by the wireless channel. For example, the code $\{1,1,1,1\}$ is inseparable with the code $\{-1,-1,-1,-1\}$ rotated by $\pi$ radian, so that the number of code sets which we can distinguish are $256 / 4=64$. Such 64 correlation results are assigned to 64 states, and we call them as correlation metric (CM). Eq. (1) describes all possible code sets which can be transmitted and (2) describes the correlated results of the received signal $r$ with all possible code sets.

$$
\begin{aligned}
& S_{i, k}=\left\{\begin{array}{cc}
1 & \text { if } k=0 \\
j^{\text {floor }(i / 16)} & \text { if } k=1 \\
j^{\text {floor }(i / 4)} & \text { if } k=2 \\
j^{i} & \text { if } k=3
\end{array}, \text { where } j=\sqrt{-1}\right. \\
& C M_{i, j}=\sum_{k=0}^{3} S_{i, k}^{*} \cdot r_{j, k}
\end{aligned}
$$

where $i$ is a state number, $j$ is a stage number, $k$ is a chirp number, $S$ is reference signal, and $r$ is received signal.

The objective of CM is not in getting direct biorthogonal coding gain, but in mapping code energy to a value as the first step to get the full coding gain.

\section{B. Branch metric (BM)}

The correlated values, $\mathrm{CMs}$, of $i$-th stage are multiplied by that of (i-1)-th stage in order to get differential phase between $i$-th and $(i-1)$-th stages. Because the branches which connect the states of $(i-1)$ th stage and those of $i$-th stage can be linked when the differential codes between the (i-1)-th stage codes and the $i$-th stage codes are in the bi-orthogonal code table $\boldsymbol{C}$, the branch metric (BM) can be calculated according to (3) and (4). Because $I_{j, k}$ in (3) can pick out branches which have bi-orthogonal relation, we can get the undegraded bi-orthogonal coding gain.

$$
\begin{gathered}
I_{j, k}=\left\{\begin{array}{c}
\operatorname{Re}\left\{S_{j, 0} \cdot S_{k, 0}^{*}, S_{j, 1} \cdot S_{k, 1}^{*}, S_{j, 2} \cdot S_{k, 2}^{*}, S_{j, 3} \cdot S_{k, 3}^{*}\right\} \in \mathbf{C} \\
\text { and } \operatorname{Im}\left\{S_{j, 0} \cdot S_{k, 0}^{*}, S_{j, 1} \cdot S_{k, 1}^{*}, S_{j, 2} \cdot S_{k, 2}^{*}, S_{j, 3} \cdot S_{k, 3}^{*}\right\} \in \mathbf{C} \\
0, \quad \text { else }
\end{array}\right. \\
B M_{i, j, k}=C M_{i, j} \cdot C M_{i-1, k}^{*} \cdot I_{j, k}
\end{gathered}
$$

where $i$ is current stage number, $j$ the state number of $i$-th stage, $k$ the state number of $(i-1)$-th stage. To get rake receiver gain in multipath channel, (4) can be modified as (5).

$$
B M_{i, j, k}=\left(\sum_{l=1}^{L} C M_{i, j, l} \cdot C M_{i-1, k, l}^{*}\right) \cdot I_{j, k}
$$

where $L$ is the number of fingers, and $C M_{i, j, l}$ is an $l$-th finger's $\mathrm{CM}$ of $j$-th state in $i$-th stage.

\section{Path metric (PM)}

The selection process to find out optimal path is performed as shown in (6). The proposed algorithm selects the path whose signal energy is a maximum, because the BM has signal energy with differential phase information.

$$
P M_{i, j}=\max _{k}\left\{P M_{i-1, k}+\max \left(\left|\operatorname{re}\left(B M_{i, j, k}\right)\right|,\left|i m\left(B M_{i, j, k}\right)\right|\right)\right\}
$$

where $r e(x)$ means the real value of $x$ and $\operatorname{im}(x)$ the imaginary value of $x$. Further processes to decode bits such as tracing back are not described in this paper, because these processes do not affect system performance, the objective of this paper is the BER and the PER performance enhancements.

\section{SIMULATION RESULTS}

We now present some simulation results to allow for comparisons with the conventional approach and the coherent detection of coherently encoded bi-orthogonal code. Conventionally, DBO-CSS can be detected by the series of processes; that is, the matched filtering, the differential demodulation, the bi-orthogonal demapping and the decoding. And, in order to make a reference, the coherently encoded bi-orthogonal coding method (CEBCM) with the code table $\boldsymbol{C}$ as the model in figure 2 is simulated. Conventional detector model is shown in figure 3 .

We simulated in AWGN channel and the diffuse multipath channel [5]. We assume that the transceivers are synchronized perfectly, and that DAC and ADC have the infinite precision with each other. In PER simulation, 10,000 packets are simulated and each packet has 100Byte transmit data. Simulation result of PER performance in AWGN is shown in figure 4. As shown in 
figure 4, about $2.5 \mathrm{~dB}$ of required SNR can be reduced in the proposed method. Figure 5 shows the simulation results of the diffused channel in [5]. As we can see in these figures, the PER and the BER of the proposed algorithm is saturated at lower error rate than that of the conventional algorithm.

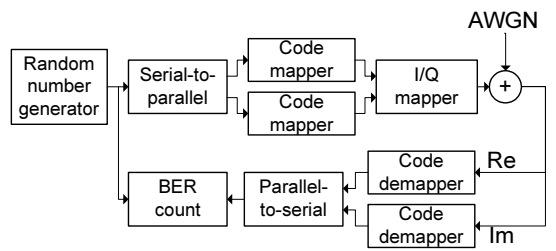

Fig. 2. Simulation model of CEBCM

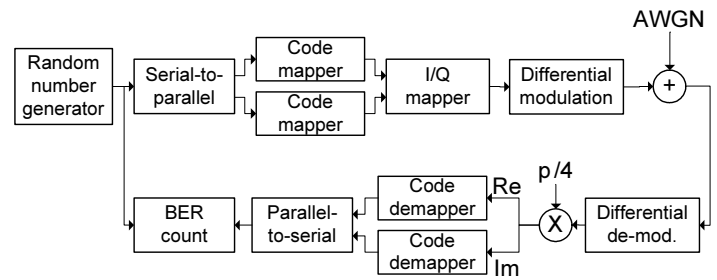

Fig. 3. Simulation model for conventional DBO-CSS detector

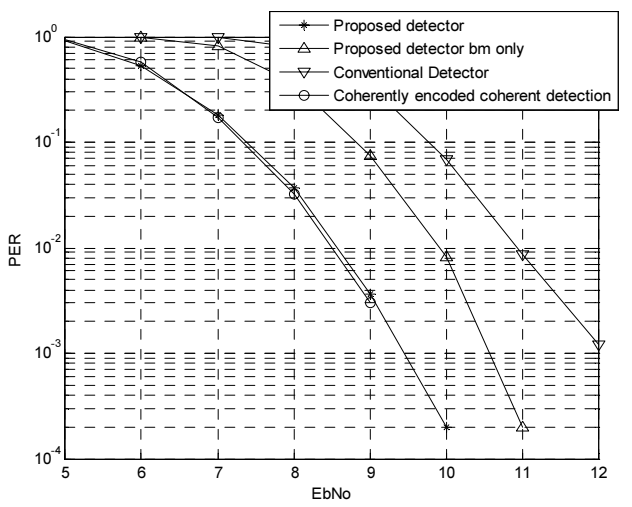

Fig. 4. PER Performances in AWGN channel

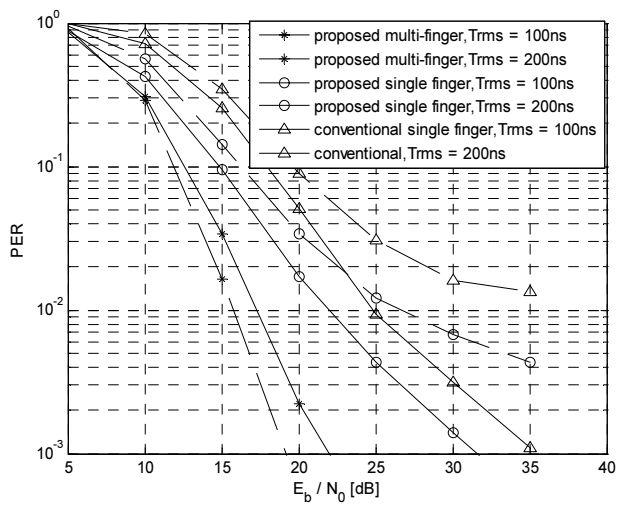

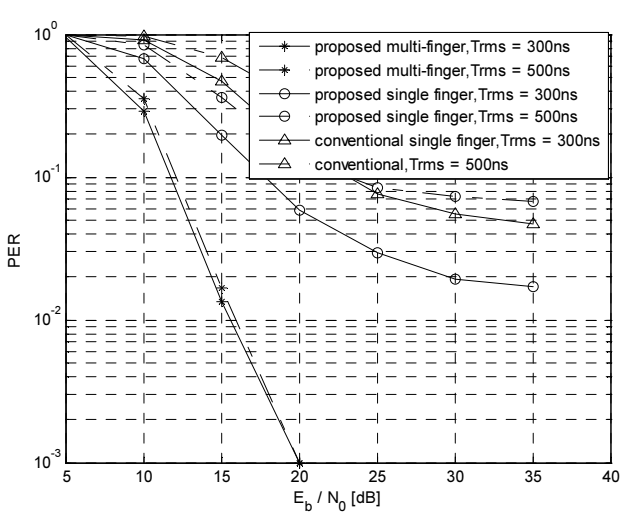

(b)

Fig. 5. Performance in diffused multipath channel (a) PER when Trms $=100$ and 200ns (b) PER when Trms $=$ 300 and 500ns

\section{CONCLUSIONS}

In this paper, we presented a new decoding algorithm for DBO-CSS which is one of the standards of IEEE802.15.4a. Frankly speaking, the hardware complexity of the proposed algorithm would be huge if we implement it on ASIC. But, if we implement the receiver by using embedded processor, the hardware complexity problem can be solved. Since the data rate of DBO-CSS is very low, the processor can afford to the computational complexity.

\section{REFERENCES}

[1] http://www.ieee802.org/15/pub/TG4a.html

[2] IEEE P802.15.4a ${ }^{\mathrm{TM}} / \mathrm{D} 5$, Sep. 2006

[3] Changkon Kim, Jiyong Yoon, Jongwha Chong, "An architecture of decision feedback differential phase detection of M-ary DPSK signals" TENCON 99, Vol. 1, pp.49-52, Sep. 1999

[4] Adachi, F., Sawahashi, M., "Decision feedback differential phase detection of M-ary DPSK signals" IEEE Transactions on Vehicular Technology, Vol. 44, pp.203-210, May 1995

[5] Perez-Jimenez, R., Berges, J., Betancor, M., "Statistical model for the impulse response on infrared indoor diffuse channels" Electronics Letters, Vol. 33, pp.1298-1300, Jul. 1997

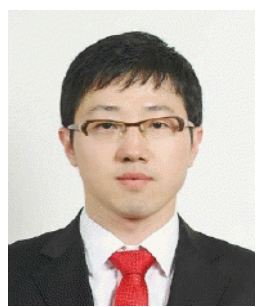

Sang-Hun Yoon received the $\mathrm{Ph}$. Degree from Hanyang University in 2008, and is currently work for Electronics and Telecommunications Research Institute in Daejeon, Korea, as a senior member of engineering staff since 2009. His research areas include ASIC design, Digital Signal Processing, Wireless Communication System Design, and Image Processing. 


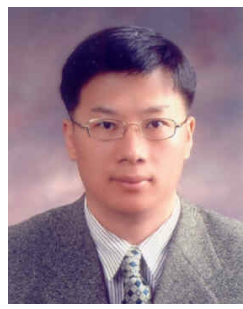

Jun-Mo Jung received the $\mathrm{Ph}$. Degree from Hanyang University in 2004, and is currently a professor at Dept. of Electronic Engineering at Kunsan National University, worked as a visiting Professor at Queensland Micro -technology Facility at Griffith University in Australia in 2009 2010. His research areas include ASIC design, Digital Signal Processing and NoC test. 